\title{
Evaluation of Dominant and Non-Dominant Hand Movements For Volleyball Action Modelling
}

Fasih Haider ${ }^{1}$, Fahim A. Salim ${ }^{2}$, Sena Busra Yengec Tasdemir ${ }^{3}$, Vahid Naghashi ${ }^{4}$, Izem Tengiz $^{5}$, Kubra Cengiz ${ }^{6}$, Dees B.W. Postma ${ }^{2}$, Robby van Delden ${ }^{2}$, Dennis Reidsma ${ }^{2}$, Bert-Jan van Beijnum ${ }^{2}$, Saturnino Luz

${ }^{1}$ The University of Edinburgh, UK, ${ }^{2}$ University of Twente, Netherlands, ${ }^{3}$ Abdullah Gul University, Turkey,

${ }^{4}$ Bilkent University, Turkey, ${ }^{5}$ Izmir University of Economics, Turkey, ${ }^{6}$ Istanbul Technical University, Turkey

\section{ABSTRACT}

In this paper, we assess the use of Inertial Measurement Units (IMU) in recognising different volleyball-specific actions. Analysis of the results suggests that all sensors in the IMU (i.e. magnetometer, accelerometer, barometer and gyroscope) contribute unique information in the classification of volleyball-specific actions. We demonstrate that while the accelerometer feature set provides the best Unweighted Average Recall (UAR) overall, "decision fusion" of the accelerometer with the magnetometer improves UAR slightly from $85.86 \%$ to $86.9 \%$. Interestingly, it is also demonstrated that the non-dominant hand provides better UAR than the dominant hand. These results are even more marked with "decision fusion".

\section{CCS CONCEPTS}

- Human-centered computing $\rightarrow$ Interactive systems and tools; • Interaction paradigms $\rightarrow$ Web-based interaction.

Permission to make digital or hard copies of part or all of this work for personal or classroom use is granted without fee provided that copies are not made or distributed for profit or commercial advantage and that copies bear this notice and the full citation on the first page. Copyrights for third-party components of this work must be honored. For all other uses, contact the owner/author(s)

ICMI '19 Adjunct, October 14-18, 2019, Suzhou, China

() 2019 Copyright held by the owner/author(s).

ACM ISBN 978-1-4503-6937-4/19/10.

https://doi.org/10.1145/3351529.3360651 


\section{Table 1: Volleyball Actions}

\begin{tabular}{l} 
Action Type \\
\hline Forearm Pass \\
One Hand Pass \\
Overhead Pass \\
Serve \\
Smash \\
Underhand Pass \\
Underhand Serve \\
Block
\end{tabular}

Table 2: Dominant Hand: Unweighted Average Recall (\%)

\begin{tabular}{llllll} 
Sensor & DT & KNN & NB & SVM & LDA \\
\hline Acc. & 70.83 & 68.83 & $\mathbf{7 9 . 8 3}$ & 59.77 & 69.56 \\
Mag. & 63.10 & 57.12 & $\mathbf{7 4 . 1 6}$ & 50.00 & 67.71 \\
Gyr. & 64.07 & 60.78 & $\mathbf{7 4 . 5 8}$ & 53.35 & 64.86 \\
Baro. & $\mathbf{5 9 . 2 2}$ & 56.53 & 57.24 & 53.01 & 56.78
\end{tabular}

Table 3: Non-Dominant Hand: Unweighted Average Recall (\%)

\begin{tabular}{llllll} 
Sensor & DT & KNN & NB & SVM & LDA \\
\hline Acc. & 71.53 & 72.98 & $\mathbf{8 3 . 9 9}$ & 66.47 & 75.90 \\
Mag. & 76.61 & 67.67 & $\mathbf{8 0 . 8 3}$ & 66.75 & 75.74 \\
Gyr. & 61.42 & 58.85 & $\mathbf{7 5 . 7 1}$ & 50.00 & 64.70 \\
Baro. & 40.86 & 38.56 & 31.53 & 50.00 & $\mathbf{5 0 . 5 3}$
\end{tabular}

\section{KEYWORDS}

Human-Media Interaction, Multimodal Feedback, Gestures Analysis

\section{ACM Reference Format:}

Fasih Haider ${ }^{1}$, Fahim A. Salim ${ }^{2}$, Sena Busra Yengec Tasdemir $^{3}$, Vahid Naghashi ${ }^{4}$, Izem Tengiz ${ }^{5}$, Kubra Cengiz $^{6}$, Dees B.W. Postma ${ }^{2}$, Robby van Delden ${ }^{2}$, Dennis Reidsma ${ }^{2}$, Bert-Jan van Beijnum ${ }^{2}$, Saturnino Luz ${ }^{1}$. 2019. Evaluation of Dominant and Non-Dominant Hand Movements For Volleyball Action Modelling. In Adjunct of the 2019 International Conference on Multimodal Interaction (ICMI '19 Adjunct), October 14-18, 2019, Suzhou, China. ACM, New York, NY, USA, 6 pages. https://doi.org/10.1145/3351529.3360651

\section{INTRODUCTION}

Automatically identifying actions in sport activities is important for multiple reasons, therefore there have been numerous studies to identify actions in sports [1,12,13,15]. Wearable devices such as Inertial Measurement Units (IMUs) $[2,14]$ are becoming increasingly popular for sports related action analysis because of their reasonable price as well as portability [13]. While researchers have proposed different configurations in terms of number and placement of sensors [17], it is ideal to keep the number of sensors to a minimum due to cost, setup effort and player's comfort $[3,6,16,17]$.

In studies involving volleyball, researchers often opted to place a sensor on the dominant arm of the player [5, 9] which seems like a reasonable choice. However the role of the non-dominant hand has not been properly explored. In this study, we have used IMU sensors on both dominant and non-dominant wrists to evaluate their respective roles in classification of action and non-actions. It is our contention that the non-dominant hand provides important data. the data gathered by the non-dominant hand is potentially less noisy, due to the fact that during a typical training session players do all sorts of actions mostly with the dominant hand which may not be of interest for analysis purposes e.g. high fiving fellow players, dribbling the ball and picking and throwing the ball around etc. It is reasonable to assume that the dominant hand is more active during these activities thereby generating movements similar to the ones performed during actions of interest and therefore making the classification of action and non-actions difficult.

In addition to analyzing the dominant vs non-dominant hands. This paper also demonstrates the fusion of different type of sensor data for the classification task. These are interesting because researchers often prefer accelerometers over other type of sensors due to their efficient power usage [9]. The analysis provided in this paper can help researchers in deciding which types of senors to use and their placement.

\section{RELATED WORK}

Many approaches have been proposed for human activity recognition. They can be categorized into two main categories: sensor-based and vision-based. 

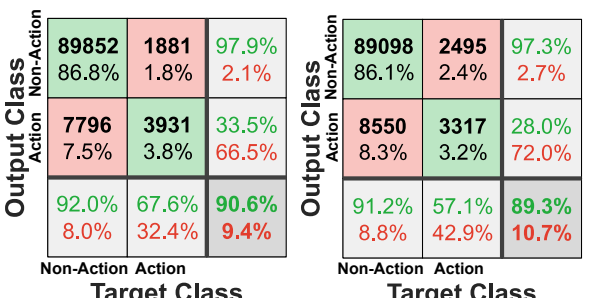

Target Class

Target Class

(a) Accelerometer

(b) Magnetometer

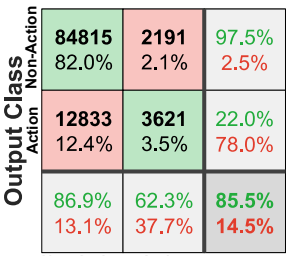

Target Class

(c) Gyroscope

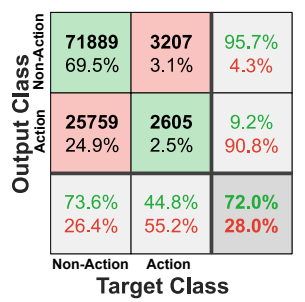

(d) Baromete
Figure 1: Confusion matrix of the best results for dominant hand along with precision, recall and overall accuracy.

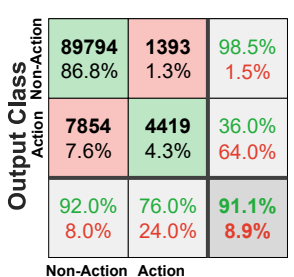

Target Class

(a) Accelerometer

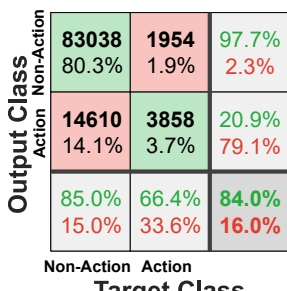

Target Class

(c) Gyroscope

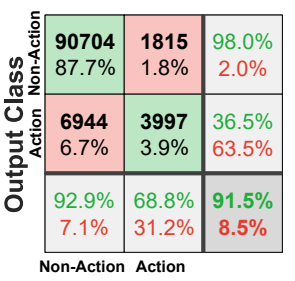

Target Class

(b) Magnetometer

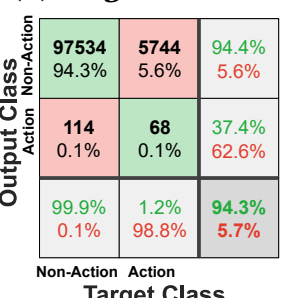

Baromete
Figure 2: Confusion matrix of the best results for non-dominant hand along with precision, recall and overall accuracy.

\section{EXPERIMENTATION}

Vision-based methods employ cameras to detect and recognize activities using several computer vision techniques. While sensor-based methods collect input signals from wearable sensors mounted on human bodies such as accelerometer and gyroscope. For example, In Liu et al. [11] identified temporal patterns among actions and used those patterns to represent activities for the purpose of automated recognition. Kautz et al. [8] presented an automatic monitoring system for beach volleyball based on wearable sensor devices which are placed at wrist of dominant hand of players. Beach volleyball serve recognition from a wrist-worn gyroscope is proposed in Cuspinera et al. [4] which is placed on the forearm of players. Kos et al. [10] proposed a method for tennis stroke detection. They used a wearable IMU device which is located on the players wrists. A robust player segmentation algorithm and novel features are extracted from video frames, and finally, classification results for different classes of tennis strokes using Hidden Markov Model are reported [18]. Jarit et al. [7] showed that the grip strength of non-dominant and dominant hands is almost the same for college baseball players.

Based on the above literature, we have concluded that the most studies take into account the role of dominant hand particularly for volleyball action modelling and the role of non-dominant hand is less explored.

Data Set: Data used in this study belongs to eight professional volleyball female players. While players were training, Inertial Measurement Unit (IMU) sensors were attached to players left and right wrists. The training session has been recorded by two different cameras which are complementing each others stage angle. Moreover, the video is annotated by three annotators using ELAN software. The list of the annotated actions is shown in Table 1.

Feature Extraction: In this study, we have used time domain features such as mean, standard deviation, median, mode, skewness and kurtosis which are extracted over a frame length of 0.5 seconds of sensor data with an overlap of $50 \%$ with the neighbouring frame. As a results we have six features for each dimension of sensor data per frame.

Classification Methods: The classification is performed using five different methods namely Decision Tree (DT, with leaf size of 5), Nearest Neighbour (KNN with $K=5$ ), Naive Bayes (NB with kernel distribution assumption), Linear Discrimination Analysis (LDA) and Support Vector Machines (SVM with a linear kernel with box constraint of 0.5 and SMO solver). The classification methods are employed in both Python and MATLAB using the statistics and machine learning toolbox in the Leave-One-Subject-Out (LOSO) cross-validation setting, where the training data do not contain any information of validation subjects. To assess the classification results, we used the Unweighted Average Recall (UAR) instead of overall accuracy as the dataset is highly imbalance. The unweighted average recall is the arithmetic average of recall of both classes. 


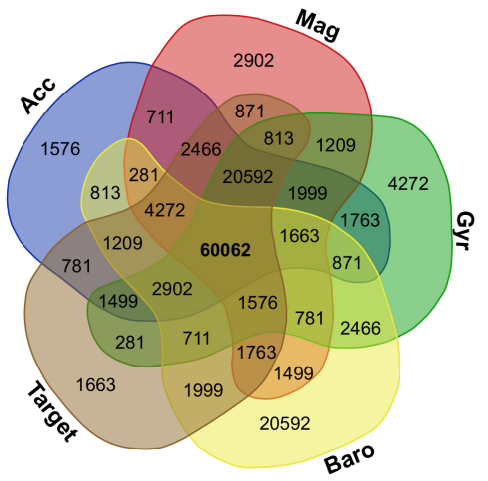

Figure 3: Venn Diagram: Dominant Hand

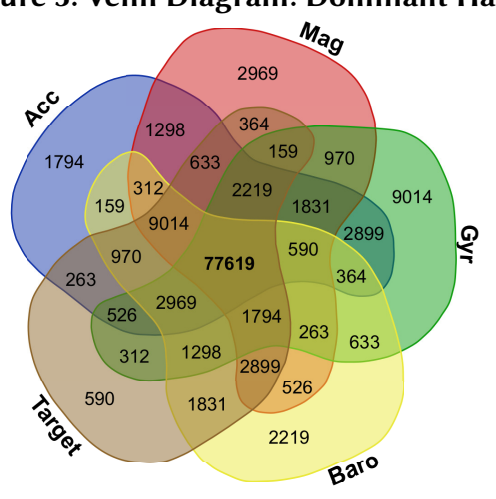

Figure 4: Venn Diagram: Non-Dominant Hand

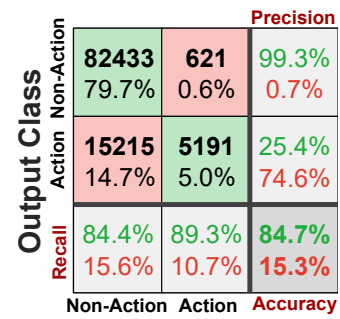

Target Class

Figure 5: Confusion matrix along with precision, recall and overall accuracy: results obtained using accelerometer and magnetometer data from both hands

\section{RESULTS AND DISCUSSION}

The UAR of dominant hand and non-dominant hand for all sensors are shown in Table 2 and Table 3 respectively. These results indicate that the non-dominant hand $(83.99 \%)$ provides better UAR than dominant hand (79.83\%), with NB being the best classifier for action detection. For further insight, the confusion matrices of the best results of each experiment (i.e. dominant hand, non-dominant hand) are also shown in Figures 1 and Figure 2 along with precision, recall and overall accuracy.

\section{Table 4: Sensor Fusion: Unweighted Average Recall (\%)}

\begin{tabular}{llll} 
Sensor & DH & NDH & Both Hands \\
\hline acc & 79.83 & 83.99 & 85.86 \\
Mag & 74.16 & 80.83 & 86.38 \\
Gyr & 74.58 & 75.71 & 81.25 \\
Acc + Mag & 81.84 & $\mathbf{8 6 . 4 2}$ & $\mathbf{8 6 . 8 7}$ \\
Acc + Gyr. & 79.40 & 83.09 & 85.02 \\
Gyr + Mag & 78.34 & 82.91 & 86.08 \\
Acc + Mag + Gyr & 80.73 & 84.58 & 85.80 \\
All & 72.91 & 84.53 & 85.32
\end{tabular}

To better understand the relationship between the different sensors, we also drew the Venn diagrams depicted in Figure 3 and Figure 4. In those Figures, the brown area (labelled "Target") represents the annotated labels, the blue area represents the predicted labels when the accelerometer feature set was used, the green area represents the predicted labels when gyroscope was used, the red ellipse represents the prediction obtained with the magnetometer feature set, and finally the yellow area represents labels predicted with the Barometer features.

The Venn diagrams suggest the information captured by different sensor is not similar, as only 60062 for dominant hand and 77619 for non-dominant hand out of 103,460 instances are detected by all the sensors. This suggests that the fusion of the results could improve overall results. We implemented a simple "decision fusion" strategy by taking a vote among all feature sets i.e fusing the output of the best classifiers for each sensor and the results are shown in Table 4. The reported UAR results are quite promising, indicating that the sensors placed at the wrist of players could be used to detect either a player is performing a volleyball action or not. It also suggest that fusion of accelerometer and magnitude sensors is providing the best UAR (as shown in Table 4 and Figure 5) when placed on both hand. However placing magnitude and accelerometer on non-dominant hand provides slightly less UAR than placing them on both hands. However, theses results need further research to investigate the different features representation (such as spectrogram) for the recognition task.

This study will also help in lowering the sensors for the players which could results in cost reduction of system and making the system less intrusive. 
The reported study is part of the Smart Sports Exercises project in which we aim to develop new forms of volleyball training using wearable sensors data and pressure sensitive in-floor displays to provide analysis and feedback in an interactive manner. While we are interested not only in action and non-action but also the type of action such as serve, forearm pass. It may be the case that dominant hand plays a crucially important role in determining the type of action. However, in many applications such as fatigue and stamina estimation [15], researchers are only interested in determining the amount of actions performed regardless of their type. In such cases, the reported results (i.e. UAR) show an interesting case of using non-dominant hand compared to the common practice of using sensor(s) on the dominant hand [5, 9].

\section{CONCLUSION}

This paper demonstrates the relevance of sensor features for volleyball action modelling. Machine learning methods operating on extracted features provide UAR of up to $86.87 \%$, well above the baseline of $50 \%$. Although dominant hand provides promising results but non-dominant hand provides better results than dominant hand and capture information which the dominant hand in missing. The outcomes of our study could be used to facilitate the development of a video tagging system for players and coaches to provide interactive feedback. In future, we aim to extend the research presented here by incorporating different frequency domain features such as spectrogram. We are also in the process of collecting an extended sensor data of volleyball players, and intend to employ the methods introduced in this paper to that data to increase the generalizability of machine learning models.

\section{ACKNOWLEDGMENTS}

This work is carried out as part of the Smart Sports Exercises project funded by ZonMw Netherlands and the European Union's Horizon 2020 research and innovation program, under the grant agreement No 769661, towards the SAAM project. Sena Busra Yengec Tasdemir, is supported by the Turkish Higher Education Council's 100/2000 PhD fellowship program.

\section{REFERENCES}

[1] Timur Bagautdinov, Alexandre Alahi, François Fleuret, Pascal Fua, and Silvio Savarese. 2017. Social scene understanding End-to-end multi-person action localization and collective activity recognition. Proceedings - 30th IEEE Conference on Computer Vision and Pattern Recognition, CVPR 2017 2017-Janua (2017), 3425-3434. https://doi.org/10.1109/CVPR.2017.365 arXiv: 1611.09078

[2] Giovanni Bellusci, Fred Dijkstra, and Per Slycke. 2018. Xsens MTw : Miniature Wireless Inertial Motion Tracker for Highly Accurate 3D Kinematic Applications. Xsens Technologies April (2018), 1-9. https://doi.org/10.13140/RG.2.2.23576.49929

[3] Jorge Cancela, Matteo Pastorino, Alexandros T. Tzallas, Markos G. Tsipouras, Giorgios Rigas, Maria T. Arredondo, and Dimitrios I. Fotiadis. 2014. Wearability assessment of a wearable system for Parkinson's disease remote monitoring based on a body area network of sensors. Sensors (Switzerland) 14, 9 (2014), 17235-17255. https://doi.org/10.3390/s140917235 
[4] L Ponce Cuspinera, Sakura Uetsuji, FJ Morales, and Daniel Roggen. 2016. Beach volleyball serve type recognition. In Proceedings of the 2016 ACM International Symposium on Wearable Computers. ACM, 44-45.

[5] L. Ponce Cuspinera, Sakura Uetsuji, F. J. Ordonez Morales, and Daniel Roggen. 2016. Beach Volleyball Serve Type Recognition. In Proceedings of the 2016 ACM International Symposium on Wearable Computers (ISWC '16). ACM, New York, NY, USA, 44-45. https://doi.org/10.1145/2971763.2971781

[6] Shariman Ismadi Ismail, Effirah Osman, Norasrudin Sulaiman, and Rahmat Adnan. 2016. Comparison between Marker-less Kinect-based and Conventional 2D Motion Analysis System on Vertical Jump Kinematic Properties Measured from Sagittal View. Proceedings of the 10th International Symposium on Computer Science in Sports (ISCSS) 392, 2007 (2016), 11-17. https://doi.org/10.1007/978-3-319-24560-7

[7] Paddy Jarit. 1991. Dominant-hand to nondominant-hand grip-strength ratios of college baseball players. Journal of Hand Therapy 4, 3 (1991), 123-126.

[8] Thomas Kautz, Benjamin H Groh, Julius Hannink, Ulf Jensen, Holger Strubberg, and Bjoern M Eskofier. 2017. Activity recognition in beach volleyball using a Deep Convolutional Neural Network. Data Mining and Knowledge Discovery 31, 6 (2017), 1678-1705.

[9] Thomas Kautz, Benjamin H. Groh, Julius Hannink, Ulf Jensen, Holger Strubberg, and Bjoern M. Eskofier. 2017. Activity recognition in beach volleyball using a Deep Convolutional Neural Network: Leveraging the potential of Deep Learning in sports. Data Mining and Knowledge Discovery 31, 6 (2017), 1678-1705. https://doi.org/10.1007/s10618-017-0495-0

[10] Marko Kos, Jernej Ženko, Damjan Vlaj, and Iztok Kramberger. 2016. Tennis stroke detection and classification using miniature wearable IMU device. In 2016 International Conference on Systems, Signals and Image Processing (IWSSIP). IEEE, $1-4$.

[11] Ye Liu, Liqiang Nie, Li Liu, and David S. Rosenblum. 2016. From Action to Activity. Neurocomput. 181, C (March 2016), 108-115. https://doi.org/10.1016/j.neucom.2015.08.096

[12] Justin Matejka, Tovi Grossman, and George Fitzmaurice. 2014. Video Lens : Rapid Playback and Exploration of Large Video Collections and Associated Metadata. In Uist. 541-550. https://doi.org/10.1145/2642918.2647366

[13] Weiping Pei, Jun Wang, Xubin Xu, Zhengwei Wu, and Xiaorong Du. 2017. An embedded 6-axis sensor based recognition for tennis stroke. 2017 IEEE International Conference on Consumer Electronics, ICCE 2017 (2017), 55-58. https://doi.org/10 1109/ICCE.2017.7889228

[14] X-io Technologies. 2019. NG-IMU. http://x-io.co.uk/ngimu/

[15] Javier Vales-Alonso, David Chaves-Dieguez, Pablo Lopez-Matencio, Juan J. Alcaraz, Francisco J. Parrado-Garcia, and F. Javier Gonzalez-Castano. 2015. SAETA: A Smart Coaching Assistant for Professional Volleyball Training. IEEE Transaction on Systems, Man, and Cybernetics: Systems 45, 8 (2015), 1138-1150. https://doi.org/10.1109/TSMC.2015.2391258

[16] T. von Marcard, B. Rosenhahn, M. J. Black, and G. Pons-Moll. 2017. Sparse Inertial Poser: Automatic 3D Human Pose Estimation from Sparse IMUs. Computer Graphics Forum 36, 2 (2017), 349-360. https://doi.org/10.1111/cgf.13131 arXiv:arXiv:1703.08014v2

[17] Yufan Wang, Yuliang Zhao, Rosa H.M. Chan, and Wen J. Li. 2018. Volleyball Skill Assessment Using a Single Wearable Micro Inertial Measurement Unit at Wrist. IEEE Access 6 (2018), 13758-13765. https://doi.org/10.1109/ACCESS.2018.2792220

[18] Z Zivkovic, Ferdinand van der Heijden, M Petkovic, and Willem Jonker. 2001. Image Segmentation and Feature Extraction for Recognizing Strokes in Tennis Game Videos. In Proc. of the ASCI. 